\title{
Analytical Inverse Kinematic Solution for Original Robotic Manipulator Construction
}

\author{
${ }^{1}$ Alexey D. Shamraev, ${ }^{2}$ Igor P. Balabanov, ${ }^{3}$ Rustem R. Ziyatdinov \\ ${ }^{1-3}$ Kazan Federal University \\ Email: shamraev.leha@mail.ru
}

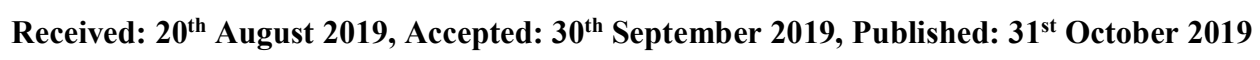

\begin{abstract}
During robot development, one of the main problems is the problem of more weight transfer to the greatest distance. The article discusses the robot kinematics, where all the movers are located as close as possible to the carrier link axis. All link engines perform rotational movements. This can significantly increase the payload of the robot. Due to kinematic relationships, the motion of one robot link is difficult to describe both in the Cartesian coordinate system and in the spherical one. The article proposes the solution to the problem of the robot intermediate link position determination, with a known position of the end unit.

We analyzed such options for the problem solution as the inverse transformation method, the analytical method, the interval method, the finite rotation and displacement method, the biquaternion solution, Mesh-based Inverse Kinematics, and FABRIK. The analytical method was optimal for the kinematics of the manipulator under consideration. Mathematical formulas are obtained that allow one to obtain the position of the intermediate links after the method application.

The resulting mathematical models will allow you to get the position of the manipulator intermediate links, to create a virtual manipulator and debug a control program on stationary computing systems. The resulting mathematical models are quite simple. Their calculation can be entrusted to a low-power computing system. This will allow the creation of autonomous adaptive systems using artificial intelligence.
\end{abstract}

\section{Keywords}

Kinematics Inverse Problem, Generalized Coordinates, Manipulator, 3 Degrees of Freedom, Analytical Method.

\section{Introduction}

During robot design, one of the main problems is the problem of more weight transfer to the greatest distance. They try to solve the restrictions imposed by the laws of physics mainly by propulsion power increase. But it is obvious that a lot of power leads to weight increase and the robot is already engaged in its own weight transfer. For example, KUKA KR AGILUS manipulators are designed in this way [1]. The second way is to design the kinematics of the manipulator in an original way. To transfer the robot weight to the center of rotation in balanced way, and spend energy on useful work. For example, ABB IRB-460 manipulators are constructed in this way [2]. The first type of robot is quite easily described by mathematical equations in a spherical coordinate system. The second type of robot is quite difficult to describe both in spherical and Cartesian coordinate system. Since we are used to think in Cartesian coordinate system, this is the way of coordinate representation that should be preferred.

The robots like IRB-460 are quite simple to design. Increasingly, such manipulators are designed and manufactured in a single copy by small companies for private use in small industries.

The manipulator design is simple. Low cost of the manipulator manufacturing and maintenance are the main advantages of these robots. But the system for the final link position calculation is complicated. At the moment, mathematical models to control the robots like IRB-460 are hidden from an average user. The user creates a robot movement program using a practical method more and more often. It outputs the end unit to the starting point and remembers the position of the intermediate units. The number of key points can reach several hundred. This way of programming a robot is extremely inefficient.

This article proposes the methodology for calculating the position of the intermediate units for the manipulator of IRB460 type.

\section{Methods}

It is necessary to solve various kinds of problems in robotics, to control the manipulation of an object in threedimensional space by the means of a manipulation device. A special place among them is occupied by the inverse kinematics problem [3], which consists in the following: let there be an absolute (fixed) rectangular Cartesian coordinate system. According to the given coordinates $(\mathrm{x}, \mathrm{y}, \mathrm{z})$ of the last link - the grip and its orientation in the absolute coordinate system - to determine generalized coordinates (q1, q2, q3, ... qn) [4], $\mathrm{n}$ is the number of degrees of the manipulator freedom. That is, to find such rotation angles (generalized coordinates) for each link of the manipulator so that its grip moves to the desired point. There are many different methods for this problem solution. All of them can be divided into two classes: exact and approximate.

Among them there are such methods as the inverse transformation method [5], the analytical method [6], the interval method [7], the finite rotation and displacement method [8], the biquaternion solution [9], Mesh-based Inverse Kinematics [10], and FABRIK [11]. 
This article may be of interest as it describes the analytical (exact) method for inverse kinematics problem solution of a nontrivial manipulator design [12]. This design involves the analytical consideration of part or all inverse kinematics problem solution.

Problem statement. The robot of the design under consideration can be seen on Fig. 1.

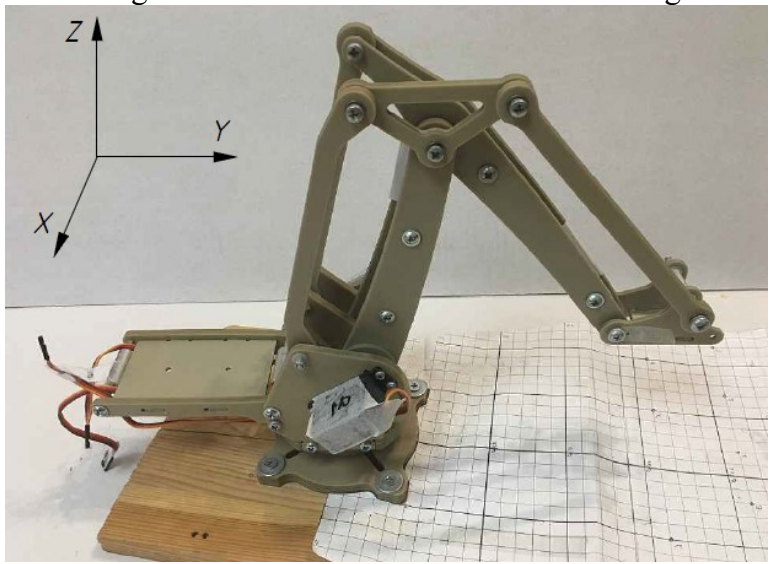

Fig. 1: Manipulator Robot

The kinematic diagram is shown on the figures 2,3 .

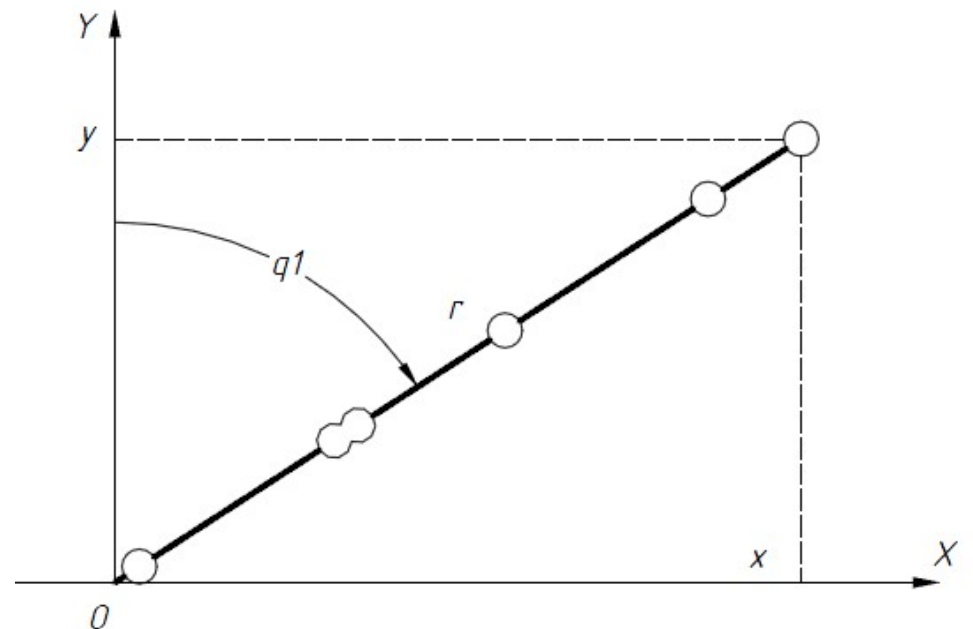

Fig. 2: Kinematic Diagram of the Robot - Top View

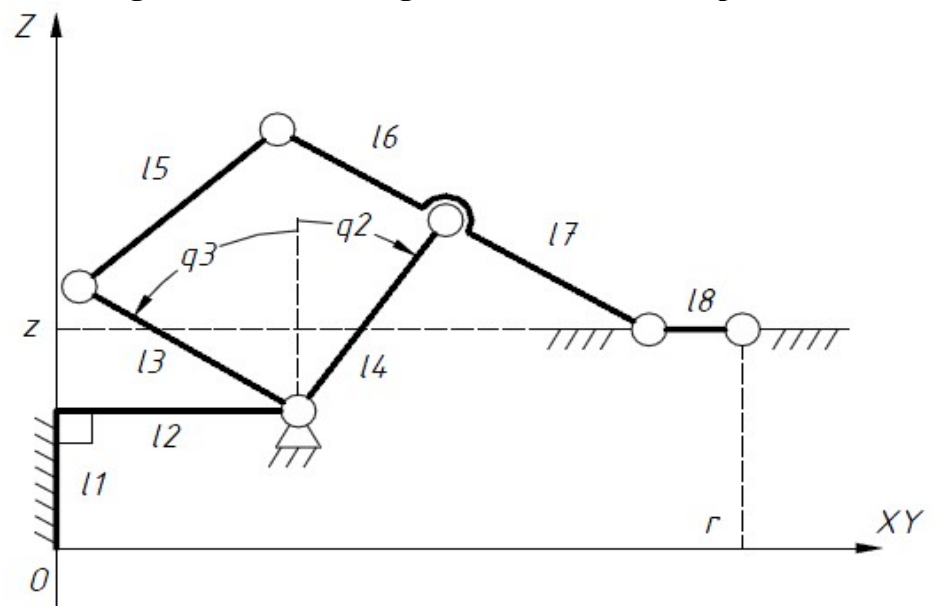

Fig. 3: Kinematic Diagram of the Robot - Left View

Let's consider this scheme. The position of the grip - the link $l_{8}$ is determined by the coordinates $(x ; y ; z)$. The link $l_{8}$, like $l_{2}$, moves parallel to the XOY plane. The beginning of the link $l_{1}$ is at the beginning of the absolute coordinate system $(X ; Y ; Z)$ and parallel to $\mathrm{Z}$ axis $[13]$. 
The robot has three generalized coordinates $\left(q_{1} ; q_{2} ; q_{3}\right)$. The angle $q_{1}$ (Fig. 2) determines the position of the grip on the XOY plane, turning the link $l_{1}$ (Fig. 3) around its axis. The angles $\left(q_{2} ; q_{3}\right)$ (Fig. 3) determine the position of the grip along the $\mathrm{Z}$ axis by turning the links $l_{4}, l_{3}$. The complexity of the solution is to convey the movement of the grip by the link $l_{3}$.

Absolute coordinates $(x ; y ; z)$ are given. It is necessary to find the generalized coordinates $\left(q_{1} ; q_{2} ; q_{3}\right)$.

The range of valid values for generalized coordinates. Let's define the range of the generalized coordinate admissible values [14]. The design of the manipulator is such that the values of the angles of each link individually can vary within the following limits:

$$
\left\{\begin{array}{l}
q_{1} \in\left(-\frac{\pi}{2} ; \frac{\pi}{2}\right) \\
q_{2} \in\left(-\frac{\pi}{4} ; \frac{\pi}{2}\right) \\
q_{3} \in\left(-\frac{\pi}{6} ; \frac{\pi}{2}\right)
\end{array}\right.
$$

However, look at fig. 3 - the links $l_{4}, l_{3}$ cannot overlap, then $q_{2}+q_{3}>0$. Finally, the range of valid values:

$$
\left\{\begin{array}{l}
q_{1} \in\left(-\frac{\pi}{2} ; \frac{\pi}{2}\right) \\
q_{2} \in\left(-\frac{\pi}{4} ; \frac{\pi}{2}\right) \\
q_{3} \in\left(-\frac{\pi}{6} ; \frac{\pi}{2}\right) \\
q_{2}+q_{3}>0
\end{array}\right.
$$

The image of permissible value range is presented on Fig. $4,\left(q_{1} ; q_{2} ; q_{3}\right)$ - on Fig. 5 .

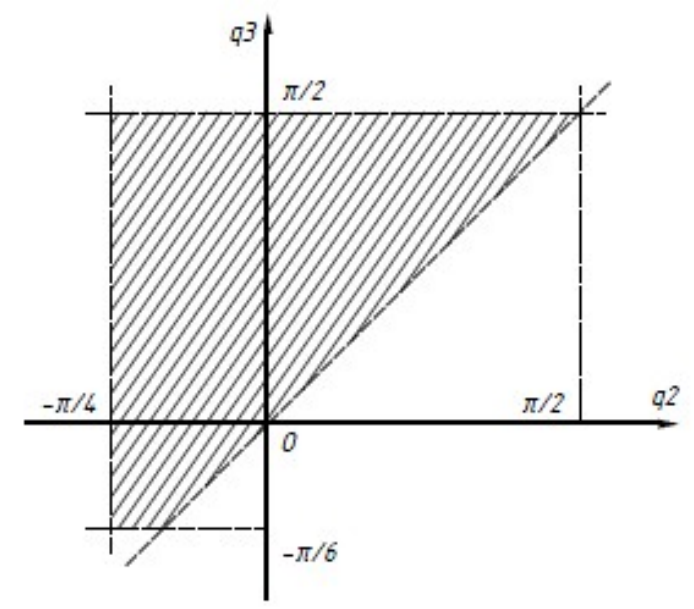

Fig. 4: The Range of Valid Values for Generalized Coordinates $\left(q_{2} ; q_{3}\right)$ 


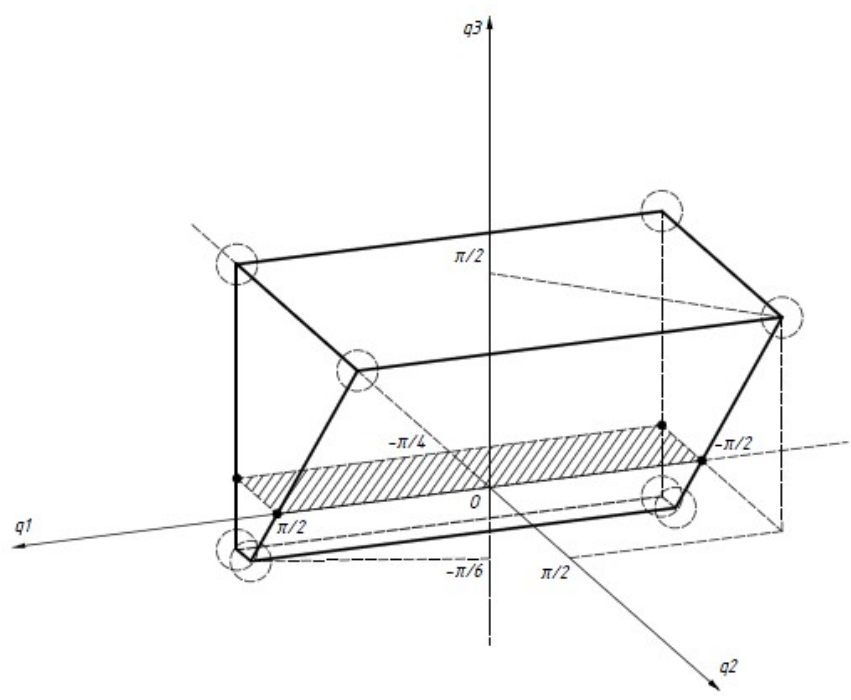

Fig. 5: The Range of Valid Values for Generalized Coordinates $\left(q_{1} ; q_{2} ; q_{3}\right)$

The analytical method for inverse kinematics problem solution. Let's proceed to solve the inverse kinematics problem [15]. Fig. 2 shows that

$$
\begin{aligned}
& q_{1}=\operatorname{arctg}\left(\frac{x}{y}\right) \\
& r=\sqrt{x^{2}+y^{2}}
\end{aligned}
$$

Also $q_{1}$ belongs to the range of valid values (1).

The angle $q_{1}$ is found, let's find the angles $\left(q_{2} ; q_{3}\right)$. Let's look on fig. 3, we will take the coordinates in this plane. $r, z$ are determined; in this plane, the links $l_{1}, l_{2}$ do not change their position; the end of the link $l_{8}$ has the coordinates $(r ; z)[16]$, then the beginning of the link has the coordinates $\left(r-l_{8} ; z\right)$. Let us introduce an additional coordinate system $(U ; V)$ [17], the beginning of which is at the junction of the links $l_{4}, l_{3}$ (Fig. 6).

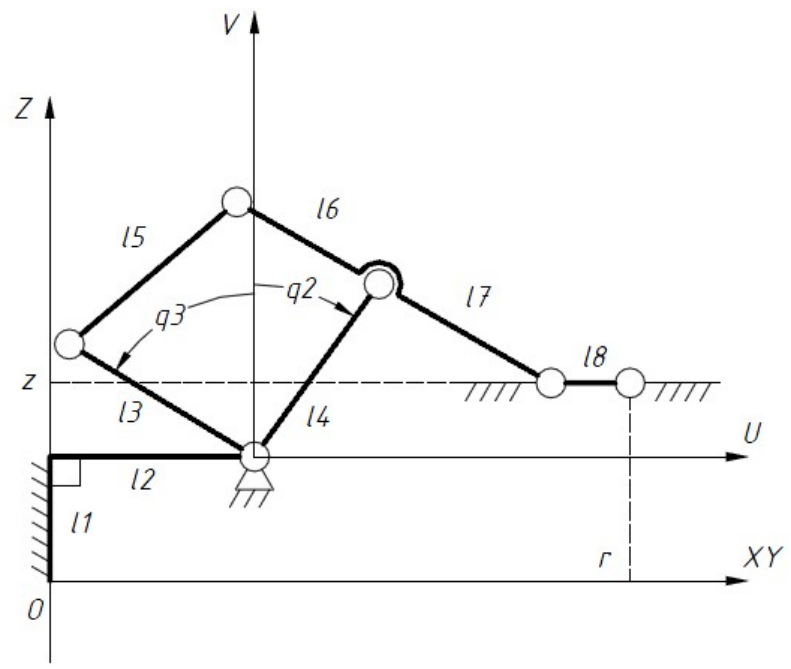

Fig. 6: Additional System of Coordinates $(U ; V)$

The transition matrix [10] into new CS: 
$T_{1}=\left(\begin{array}{ccc}1 & 0 & -l_{2} \\ 0 & 1 & -l_{1} \\ 0 & 0 & 1\end{array}\right)$

Thus,

$\left(\begin{array}{l}u \\ v \\ 1\end{array}\right)=T_{1} \cdot\left(\begin{array}{l}r \\ z \\ 1\end{array}\right)$

Let's consider the point $\left(r-l_{8} ; z\right)$, i.e.

$\left(\begin{array}{l}u \\ v \\ 1\end{array}\right)=T_{1} \cdot\left(\begin{array}{l}r-l_{8} \\ z \\ 1\end{array}\right)$

In order to find $q_{2}$ let's remove all unnecessary and leave only two links $l_{4}, l_{7}$ (Fig. 7).

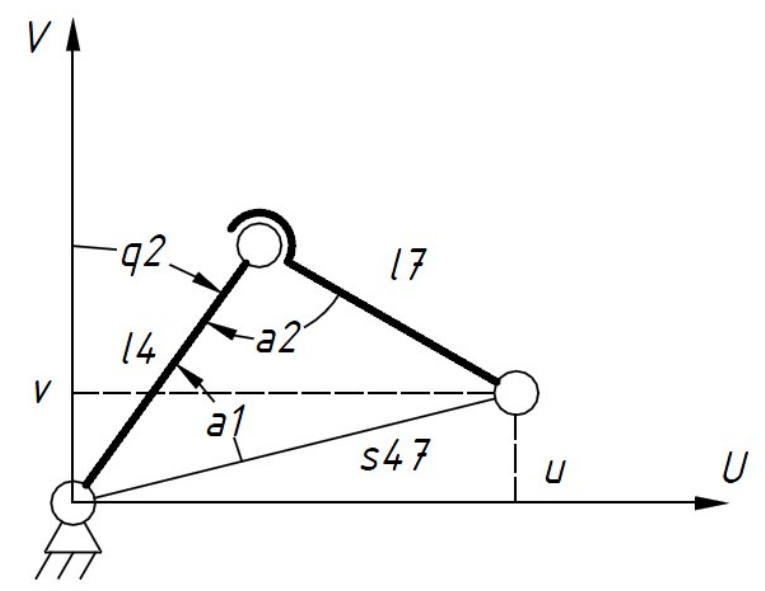

Fig. 7 : $(u ; v)$ Point Dependence on $q_{2}$ and $a_{2}$

If we connect the nodes of the links $l_{4}, l_{7}$, we get a triangle with the sides $l_{4}, l_{7}, s_{47}$, where substituting $(u ; v)$ from (5)

$$
s_{47}=\sqrt{u^{2}+v^{2}}
$$

we find the angle $a_{1}$ in this triangle. According to the cosine theorem:

$l_{7}^{2}=l_{4}^{2}+s_{47}^{2}-2 l_{4} s_{47} \cos \left(a_{1}\right)$,

Then

$a_{1}=\arccos \left(\frac{l_{4}^{2}+s_{47}^{2}-l_{7}^{2}}{2 l_{4} s_{47}}\right)$

Let's look at the Fig. 7 . The angle $a_{1}$, and the point $(u ; v)$ are determined. Then let's find $q_{2}$ :

$q_{2}=\frac{\pi}{2}-a_{1}-\operatorname{arctg}\left(\frac{v}{u}\right)$ 
The values of $a_{1}, \operatorname{arctg}\left(\frac{v}{u}\right)$ are always defined with such arguments. Therefore, $q_{2}$ satisfies the range of admissible values (1) [18-22].

Similar to $a_{1}$ let's find $a_{2}$ :

$a_{2}=\arccos \left(\frac{l_{4}^{2}+l_{7}^{2}-s_{47}^{2}}{2 l_{4} l_{7}}\right)$

Let's note that the angles $q_{2}, a_{2}$ completely determine the position of the links $l_{4}, l_{7}$. Nevertheless, one can arrive at the point $(u ; v)$ at the positions of these links symmetrically with respect to $s_{47}$. That is, there are two solutions in general, however, the earlier solution found is the only true one, since it does not turn the links [23].

In order to find the last coordinate $q_{3}$ let's remove all unnecessary (Fig. 8).

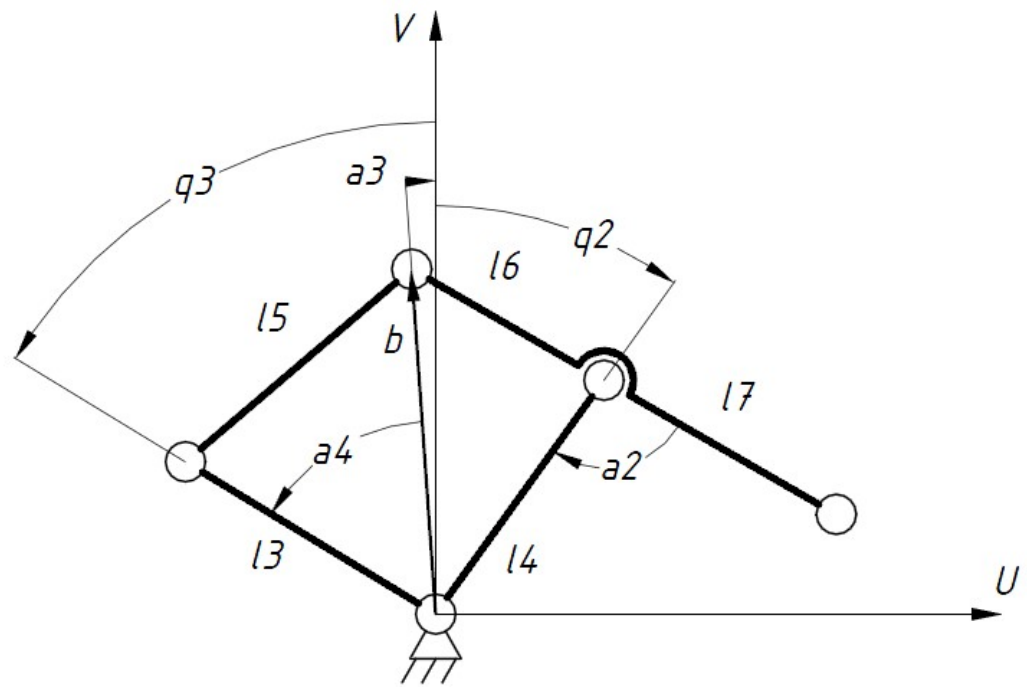

Fig. 8: The Dependence of the Angle $q_{3}$ on $a_{2}$

$q_{2}, a_{2}$ are determined. These angles completely determine the radius vector $\bar{b}$, the end of which is the connection of the links $l_{5}, l_{6}$. Let's find it:

$\bar{b}=\left(\begin{array}{l}l_{4} \cos \left(\frac{\pi}{2}-q_{2}\right)+l_{6} \cos \left(a_{2}+\frac{\pi}{2}-q_{2}\right) \\ l_{4} \sin \left(\frac{\pi}{2}-q_{2}\right)+l_{6} \sin \left(a_{2}+\frac{\pi}{2}-q_{2}\right)\end{array}\right)=\left(\begin{array}{l}u_{b} \\ v_{b}\end{array}\right)$

The angle between the radius vector $\bar{b}$ and the axis OV:

$a_{3}=\operatorname{arctg}\left(\frac{u_{b}}{v_{b}}\right)$

By the cosine theorem, the angle $a_{4}$ :

$a_{4}=\arccos \left(\frac{l_{3}^{2}+|\bar{b}|^{2}-l_{5}^{2}}{2 l_{3}|\bar{b}|}\right)$

Fig. 8 shows the following:

$q_{3}=a_{4}-a_{3}$

Also $q_{3}$ satisfies the range of valid values (1). 


\begin{abstract}
Summary
According to the obtained data, we obtain the equations to calculate the angle of controlled propulsor rotation. The most optimal will be such devices as a servo drive with feedback and the ability to hold a given angle. By moving one servo drive, at least 2 coordinates are changed. This requires a constant position recount of all the movers. This is especially required when you move the final link along a certain path. The position of the first link is determined by the formula (2). The position of the second link is determined by the formula (8). The position of the third link is written as (13).

The proposed results are exact mathematical models. But in the process of a real manipulator manufacturing, assembling and operating inaccuracies always arise. Therefore, it is necessary to introduce corrections into the equations that take into account the above inaccuracies. The most effective way is to calibrate the robot based on several key points. The more points, the more accurate model of corrections can be obtained. Calibration rules make a topic for a separate article. It should be noted that one-time calibration is enough. In the future, only significant wear of the robot units will affect the accuracy of the manipulator final unit positioning.

Let's also note that in the process of the inverse kinematics problem solution, the fact was discovered that there are several solutions for two links of this structure. However, given the restrictions imposed by other links, such as the impossibility of link overlapping, there is only one solution in the range of acceptable values.
\end{abstract}

\title{
Conclusions
}

The inverse kinematics problem of the non-trivial structure of a robot-manipulator with three degrees of freedom has been solved analytically, admissible values of generalized coordinates have been found.

\section{Acknowledgements}

The work is performed according to the Russian Government Program of Competitive Growth of Kazan Federal University.

\section{References}

[1] https://www.kuka.com/en-gb/products/robotics-systems/industrial-robots/kr-agilus

[2] https://new.abb.com/products/robotics/en/Industrial-robots/irb-460

[3] Zenkevich S.L., Yushchenko A.S. Fundamentals of manipulation robot management: Textbook for universities. M.: Publishing House of MSTU named after Bauman, 2004. - 480 p.

[4] Siciliano B. Robotics: Modelling, Planning and Control, 2009. - $632 \mathrm{p}$.

[5] Klimchik A.S., Gomolitsky R.I. et al. The development of control programs for industrial robots. Lecture course. - Minsk: Belarusian State University of Informatics and Radioelectronics, 2008. - 131 p.

[6] Borisov O.I., Gromov V.S., Pyrkin A.A. Methods for robotic application management: Textbook - St. Petersburg: ITMO University, 2016. - 108 p.

[7] Castellet A., Thomas F. Using interval methods for solving inverse kinematic problems. Proc. of the NATO Advanced Study Institute on Computational Methods in Mechanisms (NATOASI). Vol. 2. 1997, 16-28 June, Varna, Bulgaria. Varna, 1997, pp. 135-145.

[8] Danilov A.V., Kropotov A.N., Trifonov O.V. General approach to the inverse kinematics problem solution for a serial structure manipulator using finite rotation and displacement // Preprints of IAM named after M.V. Keldysh. 2018. No 81. 15 p. doi:10.20948/prepr-2018-81 URL: http://library.keldysh.ru/preprint.asp?id=2018-81

[9] Chelnokov Y. Solution to the Problems of Direct and Inverse Kinematics of the Robots-Manipulators Using Dual Matrices and Biquaternions on the Example of Stanford Robot Arm, 2015.

[10] Sumner R.W., Zwicker M., Gotsman C., Popovic J. Mesh-based inverse kinematics. ACM Transactions on Graphics (TOG) - Proceedings of ACM SIGGRAPH 2005, 2005, vol. 24, iss. 3, pp. 488-495. DOI: 10.1145/1073204.1073218

[11] Aristidou A., Lasenby J. FABRIK: A fast, iterative solver for the Inverse Kinematics problem. Graphical Models, 2011, vol. 73, iss. 5, pp. 243-260. DOI: 0.1016/j.gmod.2011.05.003

[12] Ahmadi SM, Fateh MM. Task-space control of robots using an adaptive Taylor series uncertainty estimator. International Journal of Control. 2019;92:2159-69.

[13] Ali I, Suominen O, Gotchev A, Morales ER. Methods for Simultaneous Robot-World-Hand-Eye Calibration: A Comparative Study. Sensors. 2019;19.

[14] Chembrammel P, Kesavadas T. A new implementation for online calculation of manipulator Jacobian. Plos One. 2019;14.

[15] Daneshjo N, Kralik M, Majernik M, Pajerska ED, Nascakova J. Non-collision trajectories of service industrial robots. Advances in Engineering Software. 2018;124:90-6.

[16] Del Vigo L, Peyton R, Bussi U, Oliva DE, Ieee. Computer-aided design and simulation of a robotic manipulator for educational purposes 2018 .

[17] Garriz C, Domingo R. Development of Trajectories Through the Kalman Algorithm and Application to an Industrial Robot in the Automotive Industry. Ieee Access. 2019;7:23570-8.

[18] Gupta V, Saha SK, Chaudhary H. Optimum Design of Serial Robots. Journal of Mechanical Design. $2019 ; 141$. 
[19] Hrdina J, Navrat A, Vasik P. Notes on Planar Inverse Kinematics Based on Geometric Algebra. Advances in Applied Clifford Algebras. 2018;28.

[20] Jiang P, Huang SH, Xiang J, Chen MZQ. Iteratively Successive Projection: A Novel Continuous Approach for the Task-Based Control of Redundant Robots. Ieee Access. 2019;7:25347-58.

[21] Jiang W, Yan Y, Yu LQ, Peng MH, Li HJ, Chen W. Research on dual-arm coordination motion control strategy for power cable mobile robot. Transactions of the Institute of Measurement and Control. 2019;41:3235-47.

[22] Kino H, Imamura T, Sakagami N. Error Analysis by Kinetics for Parallel-Wire Driven System Using Approximated Inverse Kinematics. Journal of Robotics and Mechatronics. 2018;30:763-71.

[23] Kobayashi Y, Harada K, Takagi K. Automatic controller generation based on dependency network of multimodal sensor variables for musculoskeletal robotic arm. Robotics and Autonomous Systems. 2019;118:55-65.

[24] Liang CJ, Lundeen KM, McGee W, Menassa CC, Lee S, Kamat VR. A vision-based marker-less pose estimation system for articulated construction robots. Automation in Construction. 2019;104:80-94.

[25] Manou E, Vosniakos GC, Matsas E. Off-line programming of an industrial robot in a virtual reality environment. International Journal of Interactive Design and Manufacturing - Ijidem. 2019;13:507-19.

[26] Singh I, Amara Y, Melingui A, Pathak PM, Merzouki R. Modeling of Continuum Manipulators Using Pythagorean Hodograph Curves. Soft Robotics. 2018;5:425-42.

[27] Wang JP, Liu SR, Zhang BT, Yu CB. Inverse kinematics-based motion planning for dual-arm robot with orientation constraints. International Journal of Advanced Robotic Systems. 2019;16.

[28] Wang J, Zhang D, Ma T, Song S, Liu W, Meng MQH, Ieee. A New Solution for the Inverse Kinematics of Concentric-Tube Robots 2018.

[29] Xu LZ, Wang WP. Natural Frequency and Modes for a Sine Movable Tooth Drive. Iranian Journal of Science and Technology-Transactions of Mechanical Engineering. 2019;43:267-73. 